\title{
Sost, independent of the non-coding enhancer ECR5, is required for bone mechanoadaptation
}

Alexander G. Robling ${ }^{1,2}$, Kyung Shin Kang ${ }^{1}$, Whitney Bullock ${ }^{1}$, Gabriela G. Loots ${ }^{3,4}$, William H. Foster ${ }^{5}$, Deepa Murugesh $^{3}$, and Damian Genetos ${ }^{5^{*}}$

Keywords: Sost; Sclerostin; mechanotransduction; ECR5; enhancer; skeleton; disuse

${ }^{1}$ Department of Anatomy \& Cell Biology, Indiana University School of Medicine, Indianapolis, IN 46202, USA.

${ }^{2}$ Department of Biomedical Engineering, Indiana University/Purdue University at Indianapolis, Indianapolis, IN 46202, USA.

${ }^{3}$ Biology and Biotechnology Division, Lawrence Livermore National Laboratory, Livermore, CA 94550, USA.

${ }^{4}$ Molecular and Cell Biology Unit, School of Natural Sciences, University of California at Merced, Merced, CA, USA.

${ }^{5}$ Department of Anatomy, Physiology and Cell Biology, University of California Davis, Davis, CA, USA.

*Authors for correspondence (e-mail: dgenetos@ucdavis.edu) 


\section{ABSTRACT}

Sclerostin (Sost) is a negative regulator of bone formation that acts upon the Wnt signaling pathway. Sost is mechanically regulated at both mRNA and protein level such that loading represses and unloading enhances Sost expression, in osteocytes and in circulation. The non-coding evolutionarily conserved enhancer ECR5 has been previously reported as a transcriptional regulatory element required for modulating Sost expression in osteocytes. Here we explored the mechanisms by which ECR5, or several other putative transcriptional enhancers regulate Sost expression, in response to mechanical stimulation. We found that in vivo ulna loading is equally osteoanabolic in wildtype and Sost $t^{--}$mice, although Sost is required for proper distribution of loadinduced bone formation to regions of high strain. Using Luciferase reporters carrying the ECR5 non-coding enhancer and heterologous or homologous hSOST promoters, we found that ECR5 is mechanosensitive in vitro and that ECR5-driven Luciferase activity decreases in osteoblasts exposed to oscillatory fluid flow. Yet, $E C R 5^{--}$mice showed similar magnitude of load-induced bone formation and similar periosteal distribution of bone formation to high-strain regions compared to wildtype mice. Further, we found that in contrast to Sost ${ }^{-1}$ mice, which are resistant to disuse-induced bone loss, $E C R 5^{-/}$mice lose bone upon unloading to a degree similar to wildtype control mice. ECR5 deletion did not abrogate positive effects of unloading on Sost, suggesting that additional transcriptional regulators and regulatory elements contribute to load-induced regulation of Sost. 


\section{INTRODUCTION}

Mechanical signals are an important factor in shaping the skeleton during development, growth and maintenance. Reduced mechanical stress or unloading, leads to significant bone loss, while increased mechanical stress or loading, causes an increase in bone mass. It was originally hypothesized that the osteocyte is the primary cell type in bone tissue that senses strain. Mechanically perturbed osteocytes produce secreted molecules that ultimately modulate the activity of osteoblasts and osteoclasts on the bone surfaces. One of the key mechanosensitive osteocyte products is the Lrp5/6 antagonist sclerostin-the protein product of the SOST gene. In vivo loading and unloading experiments conducted in rodent models consistently yield changes in SOST/sclerostin levels in the affected limb bones, where sclerostin is significantly reduced in loaded limbs and significantly increased in limbs subjected to disuse [1]. The regulation of sclerostin during mechanotransduction has important functional consequences. For example, mice harboring a transgene that prevents SOST downregulation during mechanical loading (Dmp1-hSOST) fail to exhibit an osteogenic response to in vivo mechanical stimulation. Conversely, preventing the increase in SOST expression that normally accompanies disuse, either by deleting the gene or by inactivating the protein via antibody-mediated neutralization, protects mice from disuse-induced bone loss.

As osteocyte-derived Sost is a critical permissive factor for bone loss under disuse conditions, there is considerable interest in understanding the mechanisms that control Sost transcription, particularly since modulation of Sost levels is a critical process in fine tuning bone tissue's anabolic/catabolic responses to loading or disuse. Despite the interest in Sost function and the effect of sclerostin inhibition as an osteoanabolic agent, there are relatively few studies that identify mechanistically how Sost is transcriptionally regulated. However, clues to mechanisms of Sost regulation can be found in the "natural experiment" of the rare skeletal disorder, van Buchem's (VB) disease. VB patients exhibit very high bone mass and a near complete lack of SOST expression, yet the SOST coding sequence, intron, promoter, and UTR sequences are not mutated, i.e., are genotypically normal. The suppression of SOST in these patients is due to a $52 \mathrm{~kb}$ deletion in the intergenic region-35kb downstream of SOST-between SOST and MEOX1. We recently identified a small 255bp fragment within the 52kb VB region, designated as ECR5, that is essential for Sost expression in osteocytes in vitro. Deletion of ECR5 from the mouse genome resulted in a significant decrease in Sost transcription and a high bone mass phenotype[2]. The significance of the ECR5 sequence in Sost transcription was further highlighted in in vitro experiments, where the induction of Sost expression by transforming growth factor- $\beta$ (Tgf- $\beta$ ) was dependent upon the ECR5 enhancer rather than the proximal Sost promoter[3].

If ECR5 is necessary and sufficient for the transcriptional activation of Sost in osteocytes, and if ECR5 activity is sensitive to mechanical stimulation, then $E C R 5^{--}$and Sost $^{-/}$mice should respond similarly to loading and unloading. Conversely, the milder HBM phenotype observed in ECR5 ${ }^{-1-}$ mice, compared to Sost ${ }^{-1-}$ mice, could implicate additional or alternative mechanisms that govern the mechanical regulation of Sost expression in bone. To evaluate these possibilities, we examined the requirement of Sost and ECR5 for in vivo loadinduced bone formation and for in vivo disuse-induced bone loss. We further conducted in vitro experiments designed to determine whether the ECR5 sequence is active during mechanical stimulation. Whereas Sost ${ }^{-1}$ mice were protected from the bone-wasting effects of mechanical disuse, ECR5 ${ }^{-/}$mice were not protected from disuse-induced bone loss. Despite exhibiting lower overall Sost expression, ECR5 ${ }^{-/}$mice were able to upregulate Sost in response to tail suspension. Ulnar loading was equally osteogenic in WT, Sost ${ }^{-1-}$, and ECR5

mice, but only Sost $^{-/}$mice exhibited a perturbed distribution of new load-induced bone formation that exhibited compromised preference for high-strain regions of the cortex. Further functional dissection of Sost's genomic domains is required to identify additional transcriptional regulatory elements that modulate Sost expression in response to mechanical stimulation. 


\section{MATERIALS AND METHODS}

Mice

This study was carried out in strict accordance with the recommendations in the Guide for the Care and Use of Laboratory Animals of the National Institutes of Health. The animal protocols were approved by the Indiana University Institutional Animal Care and Use Committee (IACUC). Generation of mice with the highbone-mass-causing Sost mutation have been described previously[4]. Briefly, Sost ${ }^{+-}$mice were engineered by replacing approximately $90 \%$ of the Sost coding sequence and all of the single intron, with a neomycinresistance cassette, via homologous recombination. Generation of mice with the high bone mass-causing ECR5 deletion mutation have been described previously [2]. Briefly, a 338-bp ECR5 region was replaced with a floxed neomycin cassette using Velocigene and homologous recombination. $E C R 5^{+/}$mice crosses were used to generate experimental $E C R 5^{--}$mice. The genetic background of the Sost mutant mice was a uniform mixture of $129 \mathrm{~S} 1 / \mathrm{SvIMJ}$ and $\mathrm{C} 57 \mathrm{BI} / 6 \mathrm{~J}$. The genetic background of the ECR5 mutant mice was C57BI/6J. Sixteen-week old mice were used for the disuse experiments. Male mice were used for the tail suspension studies (see below) and female mice were used for the Botox and ulnar loading studies. In each experiment, sample sizes for each genotype treatment included 8-10 mice per group, except for the gene expression studies, which included $n=6 /$ group.

\section{Ex vivo strain gauging, in vivo ulnar loading, and fluorochrome labeling}

When the animals reached $16 \mathrm{wk}$ of age, five mice from each strain (Sost ${ }^{+/+}$, Sost $^{/-}, E C R 5^{+/+}, E C R 5^{-/}$) were chosen at random, anesthetized, and sacrificed by cervical dislocation. Immediately after death, the right forearm was minimally dissected to expose the medial surface of the midshaft ulna. A single element strain gauge (EA-06-015DJ-120; Measurements Group, Raleigh, NC, USA) was bonded to the exposed medial ulnar surface at midshaft. Once fitted with a strain gauge, the forearm was loaded in cyclic axial compression using an electromagnetic actuator with feedback control. Using a $2-\mathrm{Hz}$ haversine waveform, the forearms were loaded at 1.17, 1.40, 1.62, 1.85, and $2.07 \mathrm{~N}$, during which voltage output from the strain gauge was recorded on a digital oscilloscope. Voltage measurements were converted to strain using a calibration factor derived from measured and calculated (using beam theory) strains collected from an aluminum cantilever. Strain per unit force $\left(\right.$ Sost $^{+/}=920 \mu \varepsilon / \mathrm{N} ;$ Sost $\left.^{--}=525 \mu \varepsilon / \mathrm{N} ; E C R 5^{+/+}=955 \mu \varepsilon / \mathrm{N} ; E C R 5^{/-}=915 \mu \varepsilon / \mathrm{N}\right)$ was used to adjust peak loads to deliver equal strain stimulus among the three load groups.

At 16 weeks of age, 30 female mice from each of the genotypes (Sost ${ }^{+/+}$, Sost ${ }^{/-}, \mathrm{ECR}^{+/+}$, ECR5 ${ }^{-/}$) began the ulnar loading regimen. A haversine waveform was used to apply load to the forelimb using a customized electromagnetic actuator at $2 \mathrm{~Hz}$, for 180 cycles/day. Each mouse was loaded 3dy/wk for 2 wks. Calcein (12 $\mathrm{mg} / \mathrm{kg} \mathrm{IP})$ and alizarin complexone $(20 \mathrm{mg} / \mathrm{kg} \mathrm{IP})$ were injected 5 and 14 days, respectively, after the last first bout. Mice were sacrificed 8 days after the alizarin injection. At sacrifice, the left (unloaded) and right (loaded) ulnae were dissected, cleaned and fixed in $10 \%$ NBF for 2 days followed by storage in $70 \%$ ethanol at $4^{\circ} \mathrm{C}$.

\section{Quantitative histomorphometry}

The fixed ulnas were dehydrated in graded ethanols, cleared in xylene, and embedded in methylmethacrylate. Thick sections were collected at 1 to $1.5 \mathrm{~mm}$ distal to the midshaft using a diamondembedded wafering saw. Sections were ground and polished to $\sim 30 \mu \mathrm{m}$, mounted and coverslipped, then digitally imaged on a fluorescent microscope. Periosteal bone formation parameters were calculated by measuring the extent of unlabeled perimeter, single-labeled perimeter (sL.Pm), double-labeled perimeter (dL.Pm), and the area between the double labeling with Image-Pro software (MediaCybernetics Inc., Gaithersburg, MD). Derived histomorphometric parameters were calculated using standard procedure, which are mineralizing surface over bone surface $(\mathrm{MS} / \mathrm{BS}=((0.5 \times \mathrm{sL} . \mathrm{Pm}+\mathrm{dL} . \mathrm{Pm}) /$ total perimeter $\times 100)(\%))$, mineral apposition rate (MAR $=$ double labeled area $/ \mathrm{dL} . P \mathrm{~m} / 8$ days) $(\mu \mathrm{m} /$ day)), and bone formation rate $(B F R / B S=M A R \times M S / B S \times 3.65)\left(\mu m^{3} / \mu m^{2} /\right.$ year $\left.)\right)$. Relative load-induced bone formation parameters were calculated by subtracting the control arm value from the loaded arm value for each mouse. For sectoral analysis of regional bone formation along the periosteal surface, an "H" shaped mask was created in ImagePro and superimposed onto each rotated ulnar section image (see Figure 2). Labeling parameters were remeasured within the boundary of each sector, then summarized across opposite cortices (caudal with cranial, medial with lateral). 
UMR106.1 rat mature osteoblasts were seeded onto $75 \times 38 \times 1.0 \mathrm{~mm}$ glass slides $(400,000$ cells $/$ slide $)$. The next day, cells were co-transfected with 500ng Renilla luciferase under control of the thymidine kinase promoter (pRL-TK-Luc; Promega) and $1 \mu \mathrm{g}$ of pGL3-based (Promega) Firefly luciferase reporter plasmids with $\mathrm{X}$ TremeGENE HD (Roche) in Opti-MEM (Invitrogen). The pGL3-based reporter plasmids contain the human SOST promoter only (hSOST-Luc) or three copies of the ECR5 enhancer upstream of the human SOST promoter (3xECR5-hSOST-Luc) [3,5]. Twenty-four hours after transfection, slides were placed into oscillatory fluid flow chambers in flow media (MEM supplemented with $2 \%$ fetal bovine serum, $1 \%$ penicillin-streptomycin, $20 \mathrm{mM}$ HEPES, $\mathrm{pH}$ 7.2) and were subjected to a oscillatory fluid flow with a peak shear stress of \pm 20 dynes $/ \mathrm{cm}^{2}, 1 \mathrm{~Hz}$ for 6 hours at $37^{\circ} \mathrm{C}$. Immediately after cessation of oscillatory fluid flow, cells were scraped from the slide in Passive Lysis Buffer, after which luciferase activity in recovered lysates was determined using the Dual-Luciferase Reporter Assay System (Promega) and a Turner Designs Model 20/20 Luminometer. Within each sample, firefly luciferase activity was normalized to $\mathrm{pRL}-\mathrm{TK}$ to compensate for potential variations in transfection efficiency or cell number.

\section{Dual energy $x$-ray absorptiometry (DEXA)}

In some of the experiments, whole-body in vivo DEXA scans were collected two days prior to the start of the experiment and again at sacrifice. Mice were anesthetized with isofluorane ( $2 \% @ 1.5$ liters/min) during the procedure. Lower limb bone mineral content (BMC) was measured by adjusting the region of interest box to accommodate the lower limb distal to the acetabulum.

\section{Micro-computed tomography $(\mu C T)$}

The right femur was extracted at sacrifice to use in $\mu$ CT analyses (Scanco $\mu C T$ 35) as described previously [6]. The bones were placed in $10 \%$ NBF for 2 days and then stored in $70 \%$ ethanol at $4^{\circ} \mathrm{C}$. A $2.6-\mathrm{mm}$ span of the distal femoral metaphysis was scanned on a high resolution $\mu \mathrm{CT}$ ( $\mu$ CT 35; Scanco Medical AG) at 13- $\mu \mathrm{m}$ resolution using $50-\mathrm{kV}$ peak tube potential and $151-\mathrm{ms}$ integration time to measure trabecular threedimensional morphometric properties as previously described. Bone volume fraction (BV/TV) and trabecular thickness were calculated using standard algorithms.

\section{Peripheral quantitative computed tomography ( $P Q C T$ )}

In some experiments $\mathrm{pQCT}$ scans through right proximal tibia were collected two days prior to the start of the experiment and again at sacrifice. Mice were anesthetized with isofluorane ( $2 \% @ 1.5$ liters $/ \mathrm{min})$ during the procedure. The lower limb was secured to a platform that was centered in the gantry of a Norland Stratec XCT Research SA+ pQCT (Stratec Electronics, Pforzheim, Germany). A single cross-sectional level was scanned approximately $4 \mathrm{~mm}$ distal to the proximal growth plate using a slice thickness $0.26 \mathrm{~mm}$ at a collimation of $4 \times 10^{5}$ counts $/ \mathrm{sec}$ and at a voxel size of $0.07 \mathrm{~mm}$. For each slice, x-ray source was rotated through $180^{\circ}$ of projection for 1 block. The slice through the proximal tibia includes the cortical shell and secondary spongiosa. For each slice, total volumetric bone mineral content was measured from the PQCT images. Density thresholds of 500 and $900 \mathrm{mg} / \mathrm{cm}^{3}$ were used to identify mineralized bone.

\section{Hindlimb Suspension}

Hindlimb-suspended mice were individually housed in shoebox cages and a tail harness was used to suspend the experimental mice as previously described[6]. Control mice were permitted unencumbered normal movement in their cages. Mice in the bone loss studies were suspended for a total of 24 days prior to sacrifice. ECR5 mice used for gene expression were suspended for 4 days.

\section{Statistical analysis}

For the tail suspension studies, pre-intervention and post-intervention DEXA or pQCT measurements were compared. A paired t-test was used to determine if a difference occurred between the initial and final measurements within a genotype/treatment group. The percent change of a measurement from initial to final time points for each animal was calculated and the means of these percent changes were compared using student's t-test within genotype. For non-serial comparisons, two-way ANOVAs were conducted, using mechanical stimulus and genotype as main effects, and interaction terms were tested for significance as part of the main ANOVA model. Post-hoc follow up tests were performed using Fisher's protected LSD tests. Statistical significance was taken at $p<0.05$. Data are presented as mean \pm standard error.

For in vitro experiments, each independent trial composed of samples in triplicate or quadruplicate, and trials were repeated a minimum of three independent times. Unless otherwise noted, Luciferase data were 
normalized to internal control pRL-TK, and then to vehicle control to account day-to-day transfection variability, and are presented as mean +/- SEM. 


\section{RESULTS}

Enhanced mechanical stimulation is equally osteogenic in wildtype and Sost $^{-/}$mice

We have shown that Sost is tightly regulated by the mechanical loading environment[1], and that forced transgenic expression of SOST during loading prevents load-induced bone formation[7]. Because sclerostin reduction is a key step in load-induced bone formation, we investigated whether complete deletion of Sost would alter load-induced bone formation. 16-wk-old female wildtype and Sost ${ }^{-/}$mice were subjected to ulnar loading at one of three strain magnitudes. Periosteal bone formation occurred in each genotype in a straindependent manner (Figure 1A). Relative mineralizing surface (Figure 1B), apposition rates (Figure 1C), and bone formation rates (Figure 1D) were increased in a strain-dose responsive manner in both wildtype and Sost $^{-/}$mice. No mutation-related differences in periosteal osteogenic response were noted for any of the parameters, using genotype as a main effect.

Sost is required for proper localization of new bone to surfaces experiencing high strains.

While measuring the fluorochrome-labeled sections, we noticed that the periosteal regions that experience minimal change in strain during ulnar loading (i.e., along the neutral axis) appeared more heavily labeled in Sost $^{--}$mice compared to wildtype mice (Figure 2A). To assess this difference, we went back and re-measured the histological sections using a sectoral approach, and quantified the same bone formation parameters separately in the medial, lateral, caudal, and cranial sectors. Among wildtype mice, bone formation rates were significantly greater in the high strain sectors (medial and lateral cortices) compared to Sost ${ }^{-/}$mice (Figure 2B). Further, wildtype mice exhibited significantly lower bone formation rates in the low strain sectors (caudal and cranial cortices) compared to Sost ${ }^{-/}$mice (Figure 2C).

The ECR5 enhancer is mechanosensitive in vitro

Previously, we have demonstrated that short-term (two hours) of oscillatory fluid shear stress significantly suppresses Sost mRNA expression, which subsequently recovered to baseline (static controls) levels within four hours post-fluid flow[8], suggesting that mechanical loading and unloading transcriptionally regulate Sost expression. In vivo, mechanical loading decreases Sost mRNA and sclerostin protein expression in osteocytes[1], and reductions in Sost are required for load-induced periosteal bone formation [7]. Yet, these data fail to identify whether the Sost promoter or the distal enhancer ECR5 are responsive to biophysical forces.

To determine whether the osteocyte enhancer ECR5 is mechanosensitive, we transfected UMR106.1 cells with different ECR5/SOST reporter constructs, applied fluid shear, and measured reporter activity. Exposure to fluid flow significantly increased Luciferase activity in cells transfected with SV40-Luc or hSOST-Luc compared to plasmid-matched static cells (Figure 3B). SV40-Luc and SOST-Luc constructs increased reporter activity by $43 \%$ and $79 \%$ over static controls, respectively. In contrast, cells transfected with plasmids containing ECR5, regardless of the choice of heterologous SV40 or SOST promoter, decreased Luciferase activity in response to fluid flow, compared to static cells (Figure 3B).

We examined the kinetics of fluid flow-mediated changes in reporter activity. One hour of fluid flow did not significantly influence Luciferase activity, regardless of the plasmid's regulatory sequence (Figure 3C). Instead, significant increases in Luciferase activity in SOST were observed after 3 or 6 hours of fluid flow only in cells whose plasmid contained ECR5. Altering the number of copies of ECR5 $(0,1[3]$, or 3$)$ in cells exposed to fluid flow dose-dependently decreased Luciferase activity, such that each additional copy amplified the repression (Figure 3D; Pearson correlation $r=-0.9951$ ). These results demonstrated that ECR5 element responds to mechanical load to down-regulate transgene expression.

\section{Mechanical loading increases bone formation in ECR5 ${ }^{-/-}$mice}

ECR5 deficient mice $\left(E C R 5^{--}\right)$have a high bone mass phenotype due to reduced Sost expression in osteocytes[2]. To determine whether $E C R 5^{-1}$ mice phenocopy Sost ${ }^{-/}$mice regarding their response to mechanical loading, we subjected $E C R 5^{/-}$and WT littermate mice to ulnar loading using a single, matched peak strain magnitude. Relative mineralizing surface, apposition rates, and bone formation rates were increased by loading in both $E C R 5^{/ /}$and wildtype control mice (Figure 4), but no significant genotype-related differences were found for those parameters (Figure 4B-4D). We conducted a sectoral analysis of bone 
formation rates as described earlier for Sost ${ }^{--}$mice, but no differences in high strain regions (medial and lateral cortices; Figure 4E) or low strain regions (cranial and caudal cortices; Figure 4F) were detected between genotypes. These data suggest that mechanical loading increases bone formation and localization to high strain regions independent of ECR5.

Sost deficiency prevents bone loss caused by unloading

We and others have reported that mechanical disuse increases Sost expression in vitro [9] and in vivo[1]. To evaluate whether changes in Sost expression that occur with disuse have functional consequences on bone mass, we measured the effects of tail suspension on hindlimb bone mass and structural properties in 16-wkold male Sost ${ }^{-1}$ mice (Figure 5A). Wildtype tail-suspended mice lost $\sim 20 \%$ of their initial proximal tibia bone mineral content (BMC), whereas the ground control wildtype littermates did not lose a significant amount of proximal tibia BMC over the 24 day study (i.e., BMC change was not significantly different from zero). Conversely, the same comparison among Sost ${ }^{-1}$ mice revealed that tail suspended mice did not lose a significant amount of proximal tibia BMC (change was not significantly different from zero), but the ground control Sost ${ }^{-/}$littermates gained a significant amount of BMC (Figure 5B), which resulted in a significant difference between ground control and tail suspended Sost ${ }^{-/}$groups. In the distal femur, trabecular bone volume fraction (BV/TV) and trabecular thickness (Tb.Th) were significantly reduced by tail suspension in wildtype but not Sost ${ }^{--}$mice (Figures 5A, 5C, and 5D). Regardless of genotype or mechanical intervention, mice did not gain nor lose a significant amount of body weight during the course of these experiments (Figure 5E). Similar results were observed in wildtype or Sost ${ }^{--}$mice in which neuromuscular transmission was inhibited with Botox (Supplemental Figure 1).

ECR5 deficient mice are not protected from the bone-wasting effects of disuse

Because $\mathrm{Sost}^{-/}$mice are protected from the bone-wasting effects of disuse (presumably because Sost cannot be upregulated during disuse), and considering that Sost expression is at least partially under the control of ECR5, we next asked whether deletion of ECR5 is sufficient to prevent Sost upregulation during disuse, and ultimately, prevent disuse-induced bone wasting. $E C R 5^{/-}$and $E C R 5^{+/}$mice were tail suspended or housed in ground control conditions for 24 days (for skeletal microarchitecture) or 4 days (for gene expression). Wildtype mice lost $\sim 7.5 \%$ of their proximal tibia BMC as a result of tail suspension, whereas ECR5 ${ }^{\prime-}$ mice lost $\sim 10 \%$ BMC (Figures $6 \mathrm{~A}$ and 6B). Trabecular bone volume decreased in each genotype under disuse conditions; there was a modest, statistically significant difference, in trabecular bone volume between wildtype and $E C R 5^{-/}$mice under both control and suspended conditions, yet the relative decrease in trabecular BV/TV (Figure 6C) and trabecular thickness (Figure 6D) was the same regardless of genotype, suggesting that lack of ECR5 renders a disuse bone loss phenotype similar to wildtype mice.

Having observed that Sost is required for disuse-induced bone loss and because $E C R 5^{-/}$have a significant reduction in Sost expression [2], we sought whether ECR5 deficiency affects disuse-dependent transcriptional upregulation of Sost. Wildtype or $E C R 5^{/-}$mice were subjected to 4 days of tail suspension or ground control conditions, after which femoral or tibial cortical bone RNA was isolated, purified, and analyzed for Sost expresison. Sost expression was significantly increased in both tail suspended wildtype and $E C R 5^{-/}$mice (Figure 6E), suggesting that disuse-mediated upregulation of Sost transcription is independent of the ECR5 osteocyte enhancer. 


\section{DISCUSSION}

The skeleton adapts to the demands of its mechanical environment. Although this has been appreciated for centuries, how biophysical signals translates into an adaptive response remains an unresolved field that is currently under worthy of investigation. Osteocytes are the most abundant cell in skeleton, forming a complex functional network with neighboring osteocytes as well as with cells involved in skeletal adaptation (e.g., bone lining cells, mesenchymal stem cells, osteoclast precursors). Thus, current dogma suggests that osteocytes perceive changes in applied strain and coordinate the activity of cells involved in bone adaptation. What remains incompletely understood are the cellular and molecular mechanisms involved in, and required fo, coordinating an adaptive response.

Rodents and humans lacking the Sost gene demonstrate a robust high bone mass phenotype characterized by excessive osteoblast activity, demonstrating that Sost functions to inhibit bone formation. We have previously shown that osteoanabolic mechanical loading decreases Sost expression in a straindependent manner[1] and, using a transgenic approach, that suppression of Sost is required for load-induced bone formation[7]. In vitro studies have suggested that sclerostin, through antagonizing Lrp5/Lrp6-mediated stabilization of $\beta$-catenin, directly decreases osteoprogenitor proliferation or matrix maturation and mineralization by osteoblasts [10]. In contrast, the influence of sclerostin on osteoclasts appears to be indirect, mediated through an autocrine mechanism of sclerostin on osteocytes to regulate RANKL and OPG levels[11]. Functionally, pharmacologic inhibition of sclerostin activity by in vivo administration of a neutralizing antibody increases bone mass and strength in animal models of osteoporosis[12-14], enhances fracture repair[15-17], and prevents bone loss under disuse conditions [18,19].

Less attention has been focused on understanding the cellular and molecular mechanisms involved in regulation of endogenous Sost transcription. Initial studies by Sutherland et al. demonstrated that bone morphogenetic proteins (BMPs) [20] increase Sost expression. Subsequent studies found that numerous osteotropic growth factors and hormones-including parathyroid hormone[21], prostaglandin $E_{2}[22]$, transforming growth factor-beta[3], tumor necrosis factor-alpha[23] - regulate Sost expression through either the distal enhancer or its proximal promoter, suggesting that altering Sost transcription is required for these agents to elicit skeletal effects. Deletion of a $52 \mathrm{~kb}$ element $35 \mathrm{~kb}$ downstream of the SOST gene produces the human autosomal recessive skeletal dysplastic disease van Buchem disease[24], revealing that non-coding elements contribute to SOST expression. Using cross-species sequence comparison of the 52kb element deleted in van Buchem disease, we identified an enhancer element, termed ECR5, that drives Sost expression in in vitro and developmentally[5]. Deletion of the ECR5 distal enhancer decreases osteocytic expression of Sost to create a high bone mass phenotype[2]. We have found in vitro that the effect of certain osteotropic growth factors on Sost transcription, such as transforming growth factor- $\beta$, is mediated through the ECR5 enhancer rather than the proximal Sost promoter[3].

In light of our in vitro observation that MEF2 and Smad3 mediate Sost expression via ECR5, and our in vivo results demonstrating that loss of ECR5 reduces Sost expression in osteocytes, we sought to determine whether $E C R 5^{/-}$mice respond similarly to $S o s t^{-/}$mice when challenged with a similar anabolic loading stimulus. We found no difference in overall histomorphometric parameters between wildtype and Sost ${ }^{-/}$mice across three different strains, demonstrating that the periosteal osteogenic response to loading does not require Sost. These findings are consistent with those of Tu et al., wherein reductions in Sost expression are permissive for load-induced bone formation[7], but Sost expression itself is not a priori a fundamental requirement for an osteoanabolic response to load. These results are consistent with our recent report that postnatal b-catenin deletion from Dmp1-expressing osteocytes does not attenuate periosteal load-induced bone formation [25]

Load-induced periosteal bone formation occurs normally (i.e., at wildtype levels) in the absence of Sost, though small changes in the distribution of load induced bone formation were noted when Sost was deleted. Wildtype mice demonstrate greater bone formation rates in regions of higher strain (medial and lateral cortices) compared to regions of lower strain (cranial and caudal), whereas rBFR/BS in Sost ${ }^{-/}$mice was decreased relative to wildtype mice in high strain regions but increased relative to wildtype mice in low strain regions (Figures 2A-C). We have previously demonstrated that load-induced decreases in sclerostin protein expression is very mild at low strain cranial and caudal regions compared to the more dramatic decrease 
observed in the high strain medial and lateral cortices [1], suggesting that load-induced bone formation is inversely proportional to sclerostin abundance at a local level. In the absence of Sost, however, lower strains at the cranial and caudal cortices are then permissive to initiate bone formation. Thus, a new function for Sost in the skeleton is suggested, wherein it serves a s spatial coordinating mechanism that preferentially directs new bone to high strain regions and away from low strain regions.

In vitro, our reporter construct screen suggested that the ECR5 locus is mechanosensitive, as indicated by the significant decrease in luciferase activity among fluid-sheared cells transfected with ECR5-containing plasmids, but not those transfected with the human SOST promoter or a heterologous SV40 promoter. It was therefore surprising that when we followed up on this result in vivo, we found no differences in the periosteal response to loading in $E C R 5^{/-}$mice compared to wildtype mice. Further, we did not detect the altered distribution of load-induced bone formation that was observed in loaded Sost ${ }^{-/}$mice.

We next examined whether ECR5 participates in bone loss due to conditions of disuse. Hindlimb suspension for 24 days reduced proximal tibial bone mineral content (Figure 5B) and decreased diaphyseal bone volume (Figure 5C) and trabecular thickness in wildtype (Figure 5D), but not Sost ${ }^{-1}$, mice similar to previously published reports in vivo [26]., Thus, Sost ${ }^{-1}$ mice are resistant to the catabolic effects of skeletal unloading. Similarly, inhibition of neuromuscular transmission via Botox, cause disuse-induced bone loss in wildtype but not Sost ${ }^{-/}$mice (Supplemental Figure 1). Like wildtype mice, $E C R 5^{--}$mice exposed to unloading conditions lost bone, although there was a modest, statistically significant, attenuation of the magnitude of bone loss in $E C R 5^{--}$mice compared to wildtype mice, although this likely results from increased trabecular bone volume and thickness in $E C R 5^{-\alpha}$ compared to wildtype mice prior to hindlimb suspension. Thus, relative loss of trabecular bone was similar in wildtype and $E C R 5^{-\alpha}$ mice. Similarly, Sost expression was modestly different in wildtype versus $E C R 5^{--}$mice under disuse conditions, although the relative change in Sost was the same between genotypes.

Our results demonstrate that ECR5 is not required for osteoanabolic or osteocatabolic responses to altered loading conditions. These results were unexpected as we have found that ECR5 drives Sost expression in osteocytes in vivo [2], that the ECR5 locus is mechanosensitive (Figure 3), and because ECR5 mediates responsiveness to TGF- $\beta_{1}$ [3], which is activated under loading and is required for load-induced changes in Sost expression [27]. Thus, it appears that a locus independent of ECR5 mediates skeletal mechanosensitivity. Mechanoregulation of Sost may instead occur through its proximal promoter, although we found that the human SOST promoter transiently increases under in vitro loading conditions (Figure 3B). Alternately, other evolutionarily conserved regions in the van Buchem enhancer region [5] may differentially enhance or repress Sost expression in response to daily loads versus the relatively higher loads used in this study. Nonetheless, our results demonstrate that the ECR5 osteocyte enhancer is not required for altered Sost expression under dynamic loading conditions.

Acknowledgements:

Research reported in this publication was supported by National Institute of Arthritis and Musculoskeletal and Skin Diseases of the National Institutes of Health under award numbers R01AR053237 (AGR) and R01AR064255 (DCG), and by National Institute of Diabetes and Digestive and Kidney Diseases of the National Institutes of Health under award number R01DK075730 (GGL). This work was in part performed under the auspices of the U.S. Department of Energy by Lawrence Livermore National Laboratory under Contract DEAC52-07NA27344The content is solely the responsibility of the authors and does not necessarily represent the official views of the National Institutes of Health. The funders had no role in study design, data collection and analysis, decision to publish, or preparation of the manuscript 


\section{FIGURE LEGENDS}

Figure 1. Wild-type and Sost ${ }^{-/}$mice demonstrate similar periosteal anabolic responses to mechanical loading. (A) Photomicrographs of fluorochrome-labeled ulnar midshaft sections from Sost ${ }^{+/+}$and Sost ${ }^{--}$mice $^{-}$ loaded in vivo 3 days/week for 2 weeks. Load was applied at low (1800 $\mu \varepsilon)$, medium $(2300 \mu \varepsilon)$, or high $(2800$ $\mu \varepsilon)$ peak strain. Relative mineralizing surface per unit bone surface (rMS/BS; panel B), relative mineral apposition rate (rMAR; panel C), and relative bone formation rate (rBFR/BS; panel D), measured around the entire periosteal surface, were equally and dose-responsively elevated in wild-type and Sost ${ }^{/-}$mice as a result of loading. Different letters denote statistical differences among groups. $n=9-10 /$ group.

Figure 2. Distribution of new load-induced bone formation to high and low strain regions of the ulnar periosteal surface in wild-type and Sost ${ }^{--}$mice. (A) Photomicrographs of fluorochrome-labeled ulnar sections with superimposed sectors used to partition bone formation measurements. The images reveal increased labeling along the neutral axis (caudal and cranial sectors) and decreased bone formation in the high strain sectors (medial and lateral sectors) in Sost ${ }^{-\alpha}$ mice, compared to wild-type mice (images on right side of panel $A$ are close-up views of the caudal cortex from the left panels). Quantification of bone formation rates pooled for high strain (medial and lateral) (B) and low strain (cranial and caudal) (C) regions indicate that Sost

- mice were less efficient at localizing bone to the high strain surfaces than were wild-type mice. Different letters denote statistical differences among groups. $n=9-10 /$ group.

Figure 3. The ECR5 enhancer is mechanosensitive. (A) Diagram of Luciferase plasmids under control of heterologous SV40 promoter, the 2kb human SOST promoter, in the absence of presence of three copies of ECR5. (B) Oscillatory fluid flow significantly increased Luciferase activity in cells transfected with heterologous SV40 or SOST promoter, whereas plasmids containing ECR5 decreased Luciferase activity in response to fluid flow. (C) Time course of suppression of ECR5-driven Luciferase activity. (D) Dose-dependent effect of ECR5 copy number on Luciferase activity. Different letters denote statistical differences among groups.

Figure 4. Load-induced bone formation in the absence of ECR5. (A) Photomicrographs of fluorochromelabeled ulnar midshaft sections from wild-type and $E C R 5^{--}$mice loaded in vivo, 3 days/week for 2 week. Load was applied at $2800 \mu \varepsilon$ peak strain. Relative mineralizing surface per unit bone surface (rMS/BS; panel B), relative mineral apposition rate (rMAR; panel $\mathbf{C}$ ), and relative bone formation rate (rBFR/BS; panel $\mathbf{D})$, measured around the entire periosteal surface, were equally elevated in wild-type and $E C R 5^{--}$mice as a result of loading. Quantification of bone formation rates pooled for low strain regions and for high strain regions indicate that wild-type and $\mathrm{ECR} 5^{-/-}$mice were equally efficient at localizing new bone to the high strain surfaces. Different letters denote statistical differences among groups. $n=7-9 /$ group.

Figure 5. Sost is required for bone wasting under disuse conditions. (A) Representative $\mu C T$ reconstructions of the distal femur from ground control and tail-suspended wild-type and Sost ${ }^{-1}$ mice. (B) Percent change (pre-suspension scan vs. post-suspension scan) in proximal tibia bone mineral content from ground control and tail-suspended wild-type and Sost $^{-/}$mice as measured by serial pQCT scans. Bone volume fraction (C) and trabecular thickness (D) in the distal femur metaphysis as measured by $\mu$ CT. (E) Percent change (pre-suspension vs. post-suspension) in body mass in ground control and tail-suspended wild-type and Sost $^{-1}$ mice. ${ }^{*} p<0.05$ for tail suspended mice vs genotype-matched control mice. Different letters denote statistical differences among groups. $n=8-10 /$ group.

Figure 6. ECR5 is not required for bone wasting under disuse conditions. (A) Representative $\mu C T$ reconstructions of the distal femur from ground control and tail-suspended wild-type and ECR $5^{-/}$mice. (B) Percent change (pre-suspension scan vs. post-suspension scan) in proximal tibia bone mineral content from ground control and tail-suspended wild-type and ECR5 $5^{--}$mice as measured by serial pQCT scans. Bone volume fraction $(\mathbf{C})$ and trabecular thickness $(\mathbf{D})$ in the distal femur metaphysis as measured by $\mu \mathrm{CT}$. (E) Relative expression of Sost transcript, normalized to the housekeeping gene Rplp2, in the tibia and femur cortex of ground control and tail-suspended wild-type and $E C R 5^{-1-}$ mice. Different letters denote statistical differences among groups. $n=8-10 /$ group for panels $\mathbf{B}-\mathbf{D} ; n=6 /$ group for panel $\mathbf{E}$. 


\section{REFERENCES}

[1] A.G. Robling, P.J. Niziolek, L.A. Baldridge, K.W. Condon, M.R. Allen, I. Alam, et al., Mechanical stimulation of bone in vivo reduces osteocyte expression of Sost/sclerostin, J Biol Chem. 283 (2008) 5866-5875. doi:10.1074/jbc.M705092200.

[2] N.M. Collette, D.C. Genetos, A.N. Economides, L. Xie, M. Shahnazari, W. Yao, et al., Targeted deletion of Sost distal enhancer increases bone formation and bone mass, Proc Natl Acad Sci USA. 109 (2012) 14092-14097. doi:10.1073/pnas.1207188109.

[3] G.G. Loots, H. Keller, O. Leupin, D. Murugesh, N.M. Collette, D.C. Genetos, TGF- $\beta$ regulates sclerostin expression via the ECR5 enhancer, Bone. 50 (2012) 663-669. doi:10.1016/j.bone.2011.11.016.

[4] X. Li, M.S. Ominsky, Q.-T. Niu, N. Sun, B. Daugherty, D. D'Agostin, et al., Targeted deletion of the sclerostin gene in mice results in increased bone formation and bone strength, J Bone Miner Res. 23 (2008) 860-869. doi:10.1359/jbmr.080216.

[5] G.G. Loots, M. Kneissel, H. Keller, M. Baptist, J. Chang, N.M. Collette, et al., Genomic deletion of a long-range bone enhancer misregulates sclerostin in Van Buchem disease, Genome Research. 15 (2005) 928-935. doi:10.1101/gr.3437105.

[6] P.J. Niziolek, W. Bullock, M.L. Warman, A.G. Robling, Missense Mutations in LRP5 Associated with High Bone Mass Protect the Mouse Skeleton from Disuse- and Ovariectomy-Induced Osteopenia, PLoS ONE. 10 (2015) e0140775. doi:10.1371/journal.pone.0140775.

[7] X. Tu, Y. Rhee, K. Condon, N. Bivi, M.R. Allen, D. Dwyer, et al., Sost downregulation and local Wnt signaling are required for the osteogenic response to mechanical loading, Bone. (2011). doi:10.1016/j.bone.2011.10.025.

[8] S.E. Papanicolaou, R.J. Phipps, D.P. Fyhrie, D.C. Genetos, Modulation of sclerostin expression by mechanical loading and bone morphogenetic proteins in osteogenic cells, Biorheology. 46 (2009) 389399. doi:10.3233/BIR-2009-0550.

[9] J.M. Spatz, M.N. Wein, J.H. Gooi, Y. Qu, J.L. Garr, S. Liu, et al., The Wnt-inhibitor Sclerostin is Upregulated by Mechanical Unloading in Osteocytes in-vitro, J Biol Chem. (2015) jbc.M114.628313. doi:10.1074/jbc.M114.628313.

[10] P. ten Dijke, C. Krause, D.J.J. de Gorter, C.W.G.M. Löwik, R.L. van Bezooijen, Osteocyte-derived sclerostin inhibits bone formation: its role in bone morphogenetic protein and Wnt signaling, J Bone Joint Surg Am. 90 Suppl 1 (2008) 31-35. doi:10.2106/JBJS.G.01183.

[11] A.R. Wijenayaka, M. Kogawa, H.P. Lim, L.F. Bonewald, D.M. Findlay, G.J. Atkins, Sclerostin Stimulates Osteocyte Support of Osteoclast Activity by a RANKL-Dependent Pathway, PLoS ONE. 6 (2011) e25900. doi:10.1371/journal.pone.0025900.

[12] X. Li, M.S. Ominsky, K.S. Warmington, S. Morony, J. Gong, J. Cao, et al., Sclerostin antibody treatment increases bone formation, bone mass, and bone strength in a rat model of postmenopausal osteoporosis, J Bone Miner Res. 24 (2009) 578-588. doi:10.1359/jbmr.081206.

[13] M.S. Ominsky, F. Vlasseros, J. Jolette, S.Y. Smith, B. Stouch, G. Doellgast, et al., Two doses of sclerostin antibody in cynomolgus monkeys increases bone formation, bone mineral density, and bone strength, J Bone Miner Res. 25 (2010) 948-959. doi:10.1002/jbmr.14.

[14] D. Zhang, M. Hu, T. Chu, L. Lin, J. Wang, X. Li, et al., Sclerostin antibody prevented progressive bone loss in combined ovariectomized and concurrent functional disuse, Bone. 87 (2016) 161-168. doi:10.1016/j.bone.2016.02.005.

[15] M.S. Ominsky, C. Li, X. Li, H.L. Tan, E. Lee, M. Barrero, et al., Inhibition of sclerostin by monoclonal antibody enhances bone healing and improves bone density and strength of nonfractured bones, $\mathrm{J}$ Bone Miner Res. 26 (2011) 1012-1021. doi:10.1002/jbmr.307.

[16] M.M. McDonald, A. Morse, K. Mikulec, L. Peacock, N. Yu, P.A. Baldock, et al., Inhibition of sclerostin by systemic treatment with sclerostin antibody enhances healing of proximal tibial defects in ovariectomized rats, J Orthop Res. 30 (2012) 1541-1548. doi:10.1002/jor.22109.

[17] M.U. Jawad, K.E. Fritton, T. Ma, P.-G. Ren, S.B. Goodman, H.Z. Ke, et al., Effects of sclerostin antibody on healing of a non-critical size femoral bone defect, J Orthop Res. 31 (2013) 155-163. doi:10.1002/jor.22186.

[18] J.M. Spatz, R. Ellman, A.M. Cloutier, L. Louis, M. van Vliet, L.J. Suva, et al., Sclerostin antibody inhibits skeletal deterioration due to reduced mechanical loading, J Bone Miner Res. 28 (2013) 865874. doi:10.1002/jbmr.1807. 
[19] L.A. Beggs, F. Ye, P. Ghosh, D.T. Beck, C.F. Conover, A. Balaez, et al., Sclerostin inhibition prevents spinal cord injury-induced cancellous bone loss, J Bone Miner Res. 30 (2015) 681-689. doi:10.1002/jbmr.2396.

[20] M.K. Sutherland, J.C. Geoghegan, C. Yu, D.G. Winkler, J.A. Latham, Unique regulation of SOST, the sclerosteosis gene, by BMPs and steroid hormones in human osteoblasts, Bone. 35 (2004) 448-454. doi:10.1016/j.bone.2004.04.019.

[21] H. Keller, M. Kneissel, SOST is a target gene for PTH in bone, Bone. 37 (2005) 148-158. doi:10.1016/j.bone.2005.03.018.

[22] D.C. Genetos, C.E. Yellowley, G.G. Loots, Prostaglandin E2 signals through PTGER2 to regulate sclerostin expression, PLoS ONE. 6 (2011) e17772. doi:10.1371/journal.pone.0017772.

[23] C. Vincent, D.M. Findlay, K.J. Welldon, A.R. Wijenayaka, T.S. Zheng, D.R. Haynes, et al., ProInflammatory Cytokines TNF-Related Weak Inducer of Apoptosis (TWEAK) and TNFa Induce the Mitogen-Activated Protein Kinase (MAPK)-Dependent Expression of Sclerostin in Human Osteoblasts*, J Bone Miner Res. 24 (2009) 1434-1449. doi:10.1359/jbmr.090305.

[24] W. Balemans, N. Patel, M. Ebeling, E. Van Hul, W. Wuyts, C. Lacza, et al., Identification of a $52 \mathrm{~kb}$ deletion downstream of the SOST gene in patients with van Buchem disease, J Med Genet. 39 (2002) 91-97.

[25] K.-S. Kang, J.M. Hong, A.G. Robling, Postnatal $\beta$-catenin deletion from Dmp1-expressing osteocytes/osteoblasts reduces structural adaptation to loading, but not periosteal load-induced bone formation, Bone. (2016). doi:10.1016/j.bone.2016.04.028.

[26] C. Lin, X. Jiang, Z. Dai, X. Guo, T. Weng, J. Wang, et al., Sclerostin mediates bone response to mechanical unloading through antagonizing Wnt/beta-catenin signaling, J Bone Miner Res. 24 (2009) 1651-1661. doi:10.1359/jbmr.090411.

[27] J. Nguyen, S.Y. Tang, D. Nguyen, T. Alliston, Load regulates bone formation and Sclerostin expression through a TGF $\beta$-dependent mechanism, PLoS ONE. 8 (2013) e53813.

doi:10.1371/journal.pone.0053813. 


\section{A \\ Sost $t^{+/+}$}

Sost ${ }^{-1-}$

A.

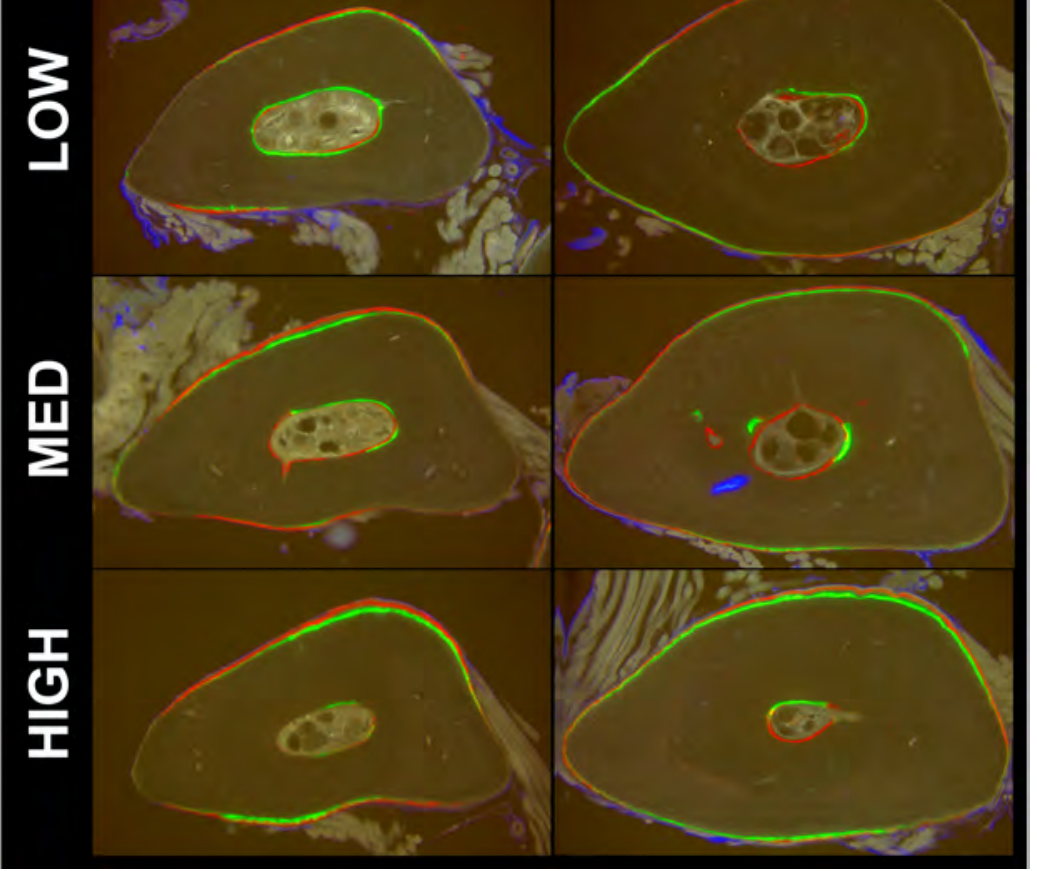

C.

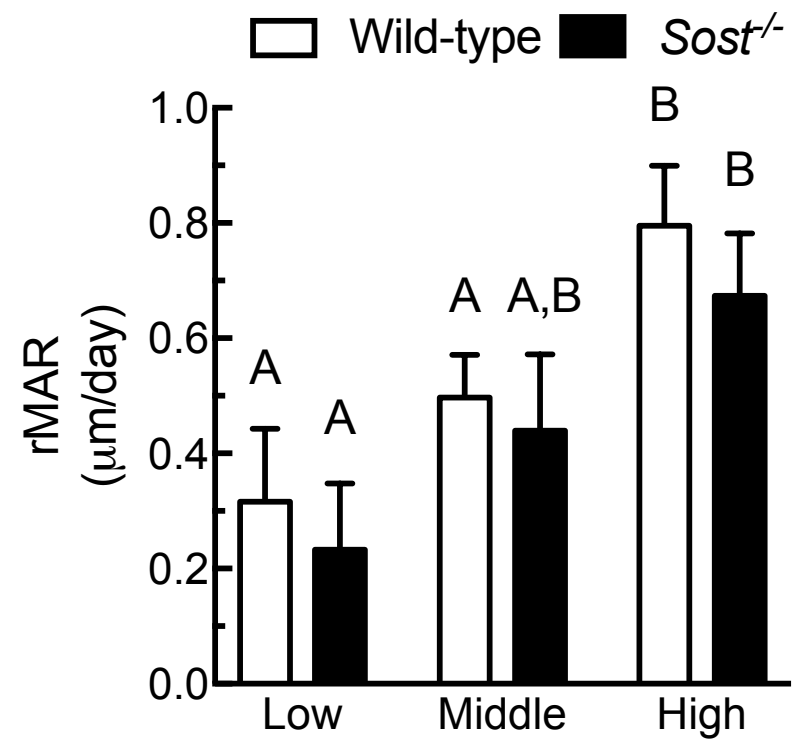

B.

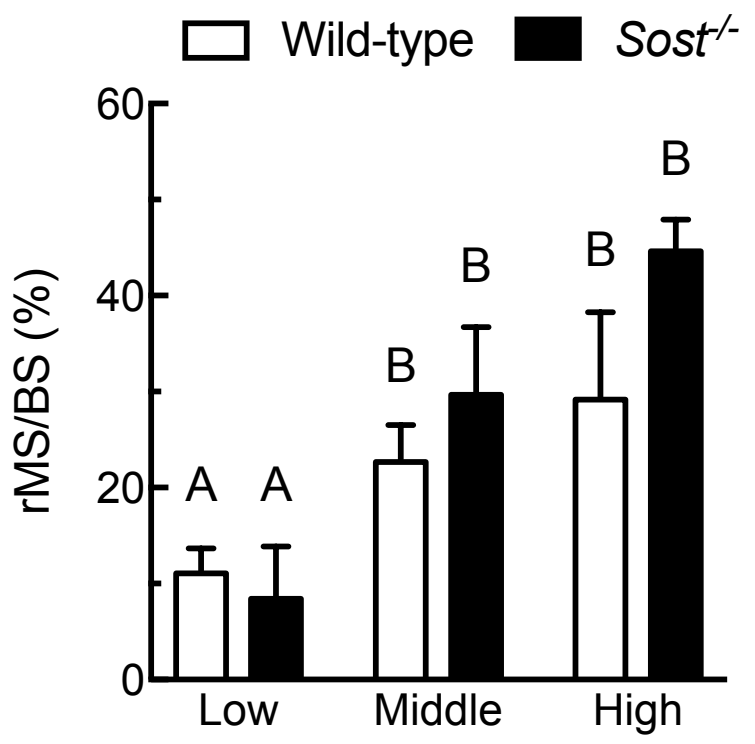

D.

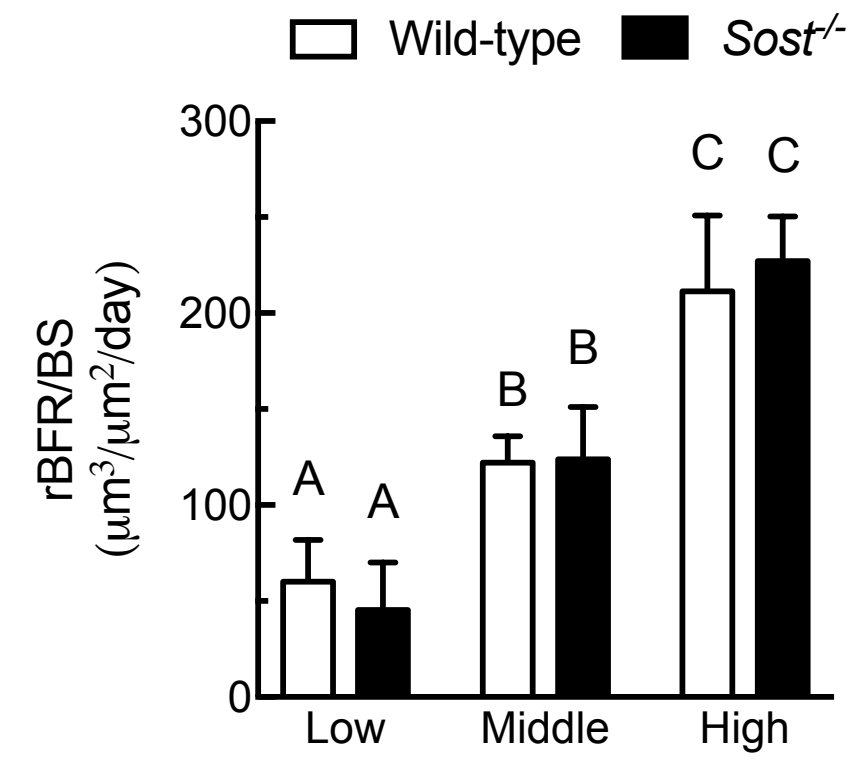


A.

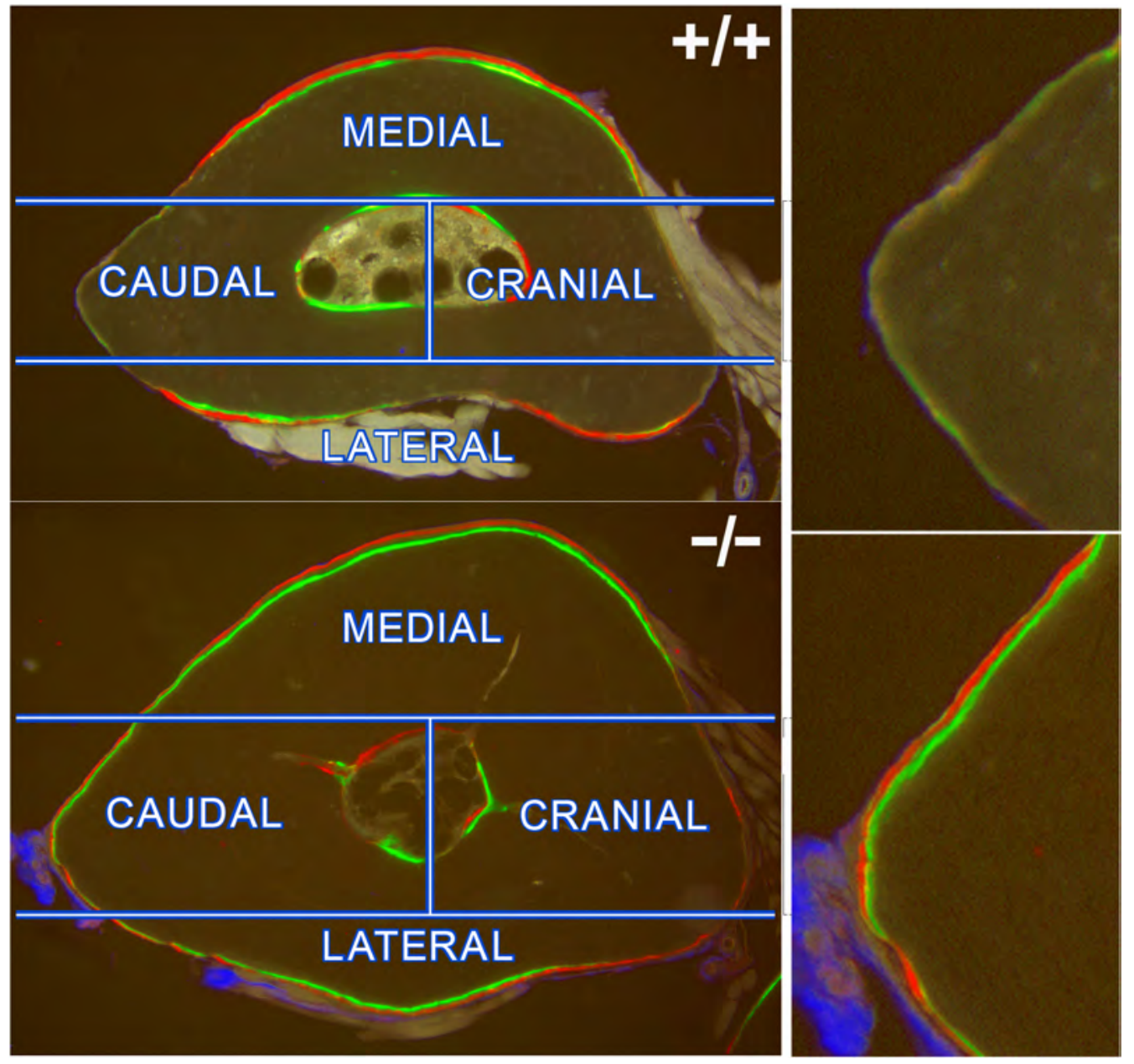

B. Medial and lateral cortices

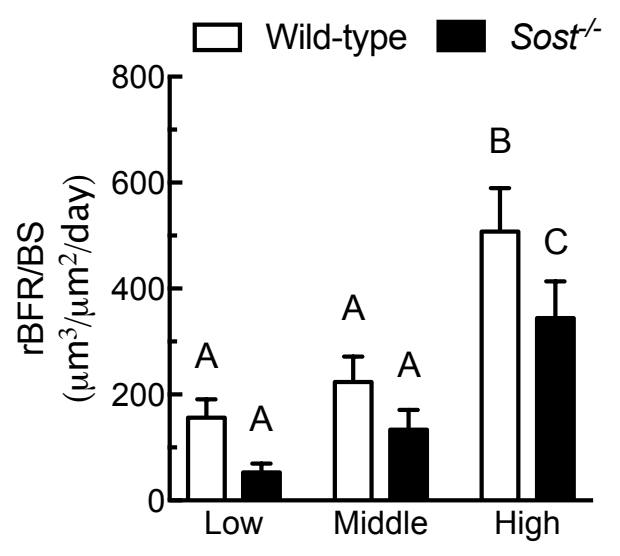

C. Cranial and caudal cortices

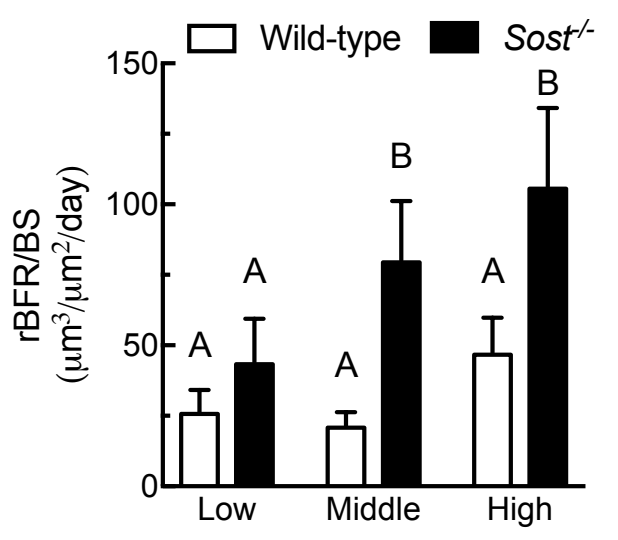


A.

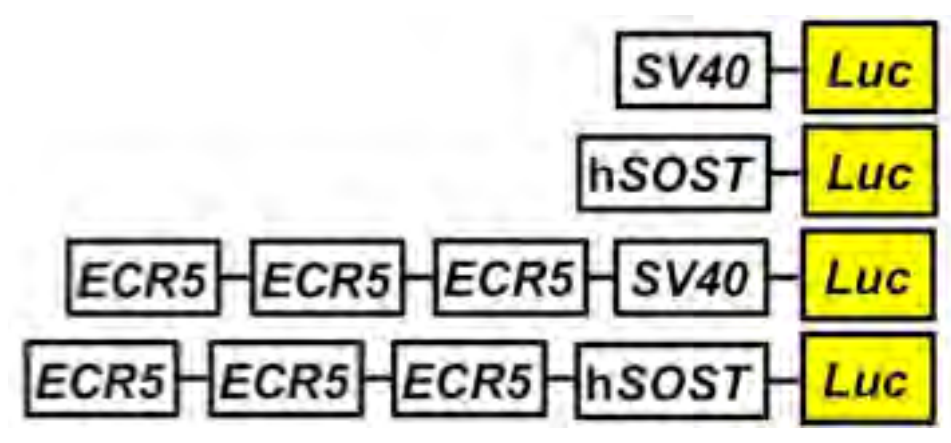

c.

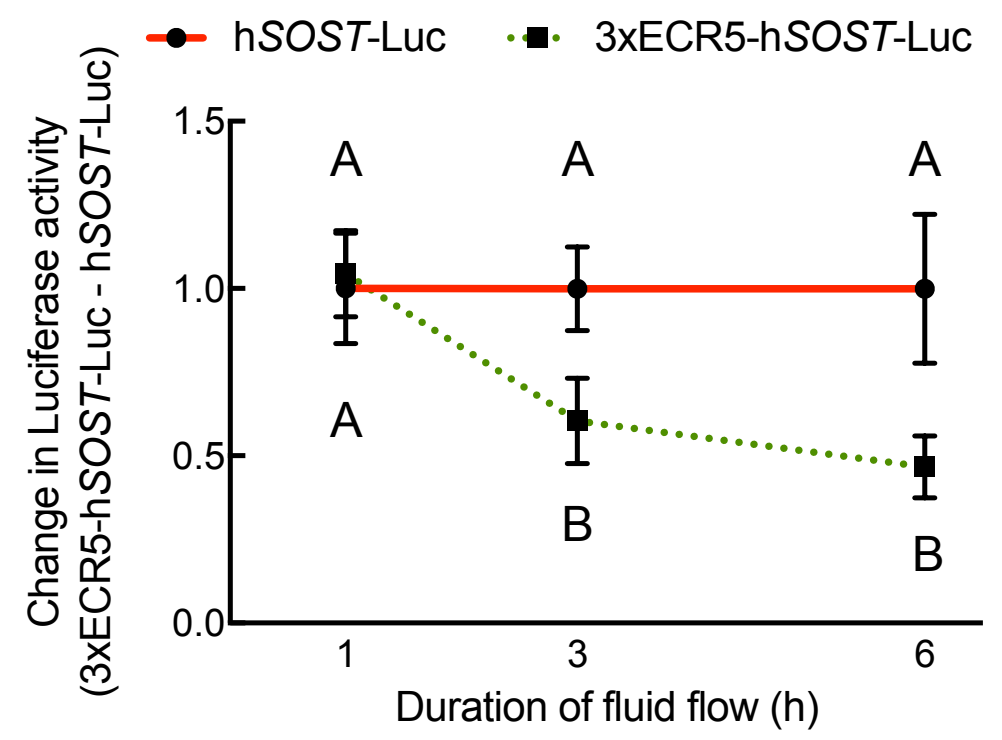

B. $\quad 2.5[\square$ Promoter only $\square+3 x$ xCR5

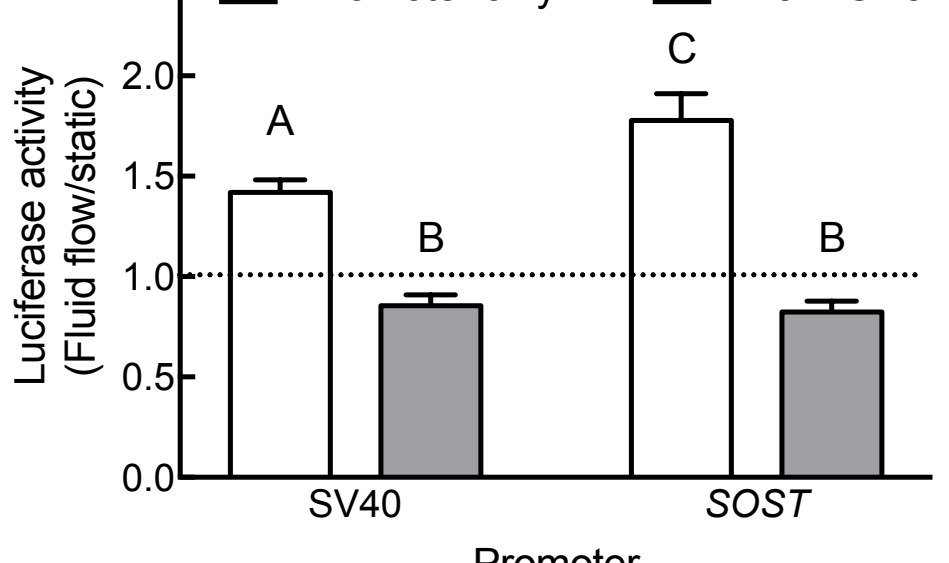

Promoter

D.

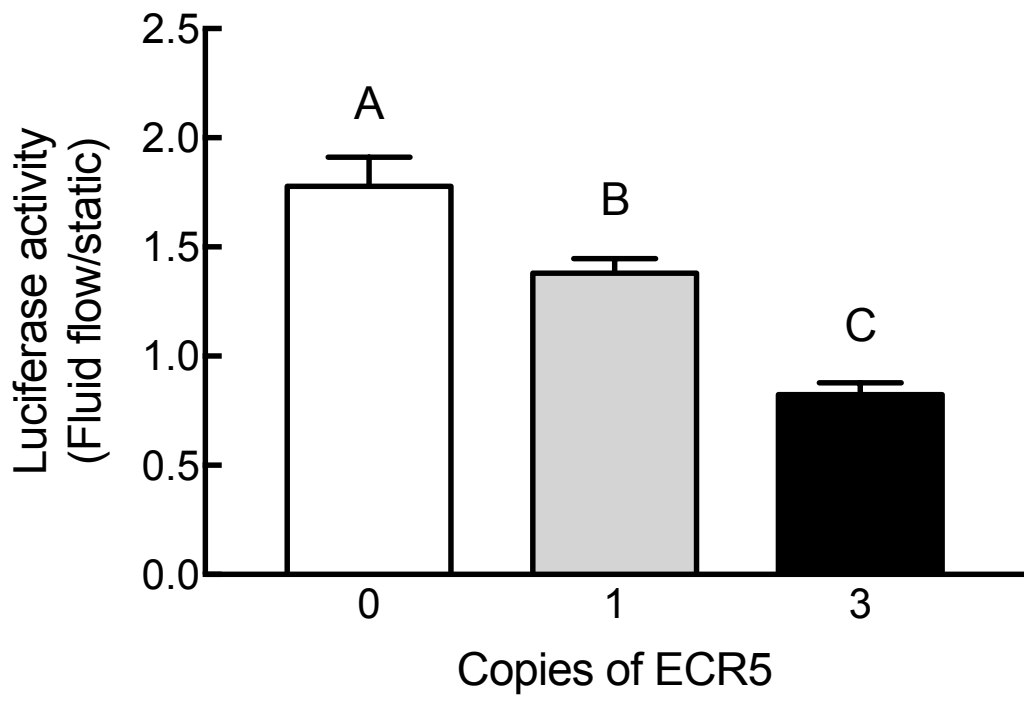


A.

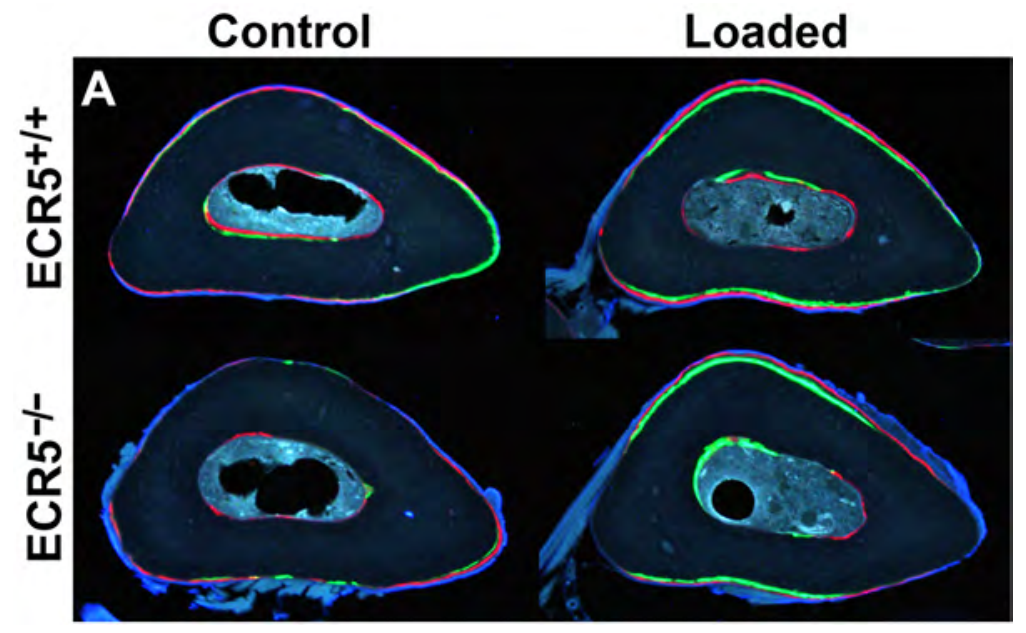

B.

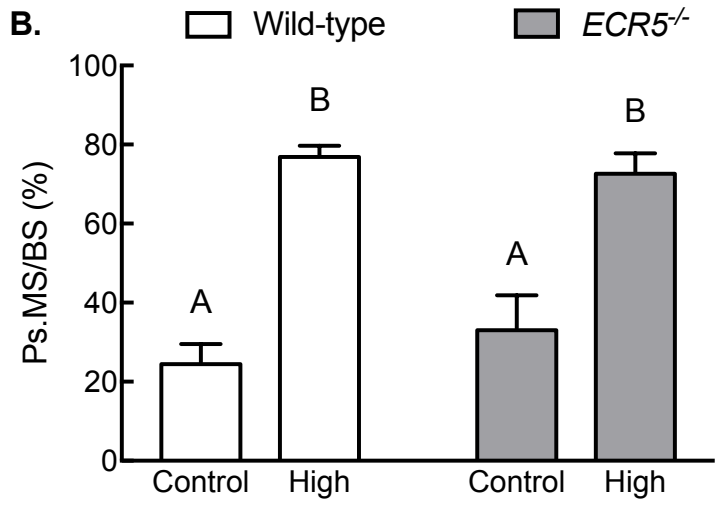

c $\square$ Wild-type $\square E C R 5^{-/}$

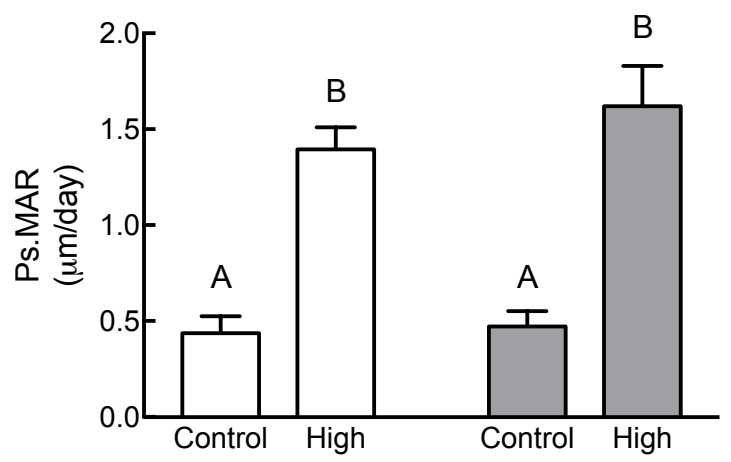

D. $\square$ Wild-type $\square$ ECR $5^{-/}$

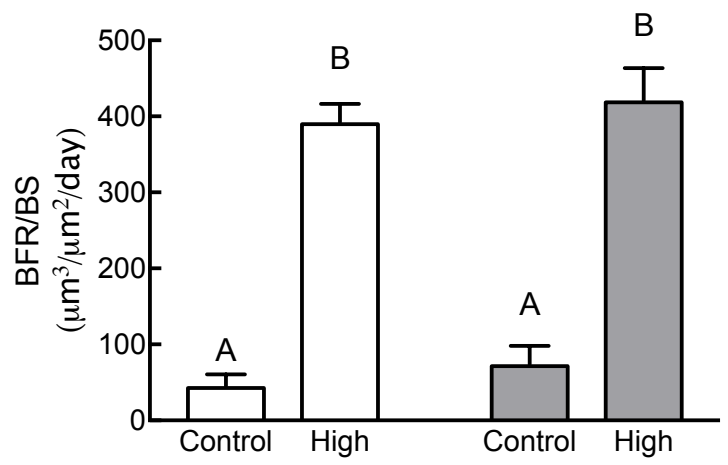

E. Medial and lateral cortices

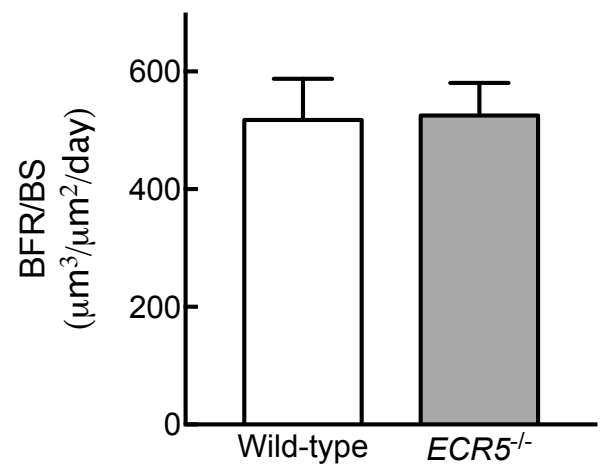

F. Cranial and caudal cortices

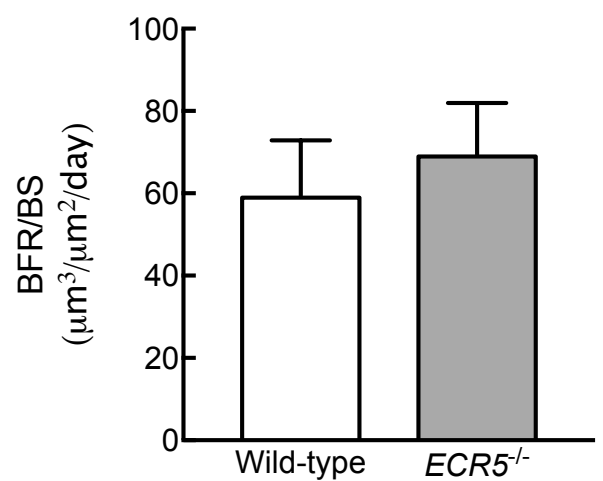


Ground Ctrl.
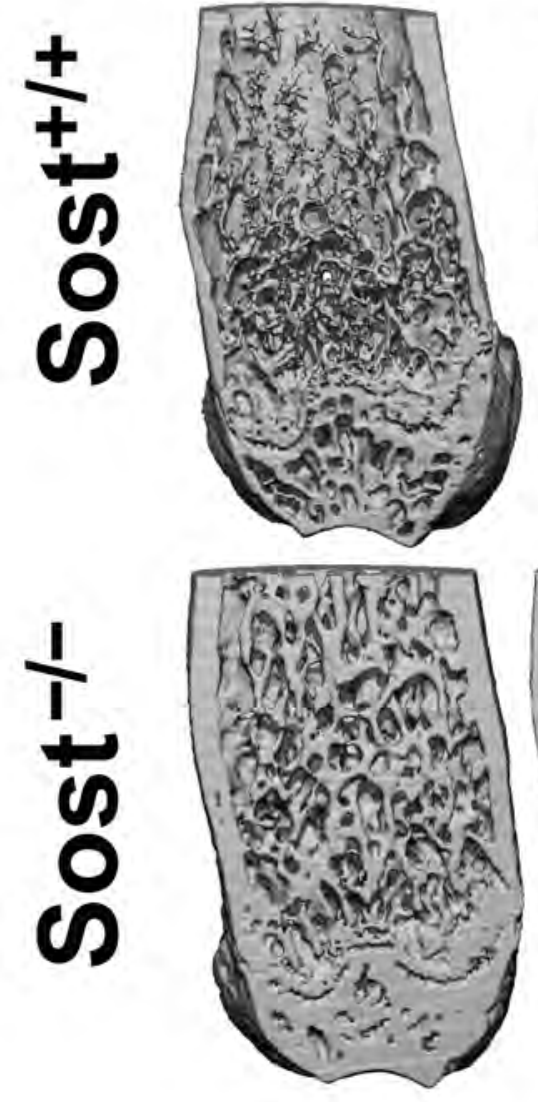

Tail Susp.
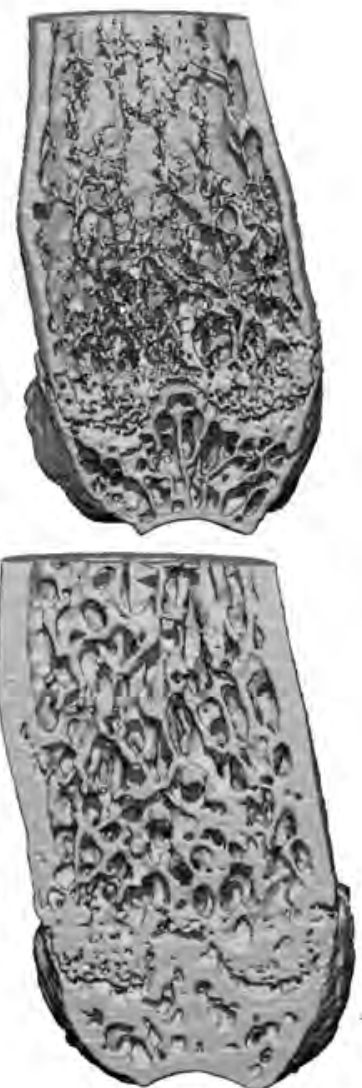

B. $\square$ Wild-type $\square$ Sost $^{-1}$

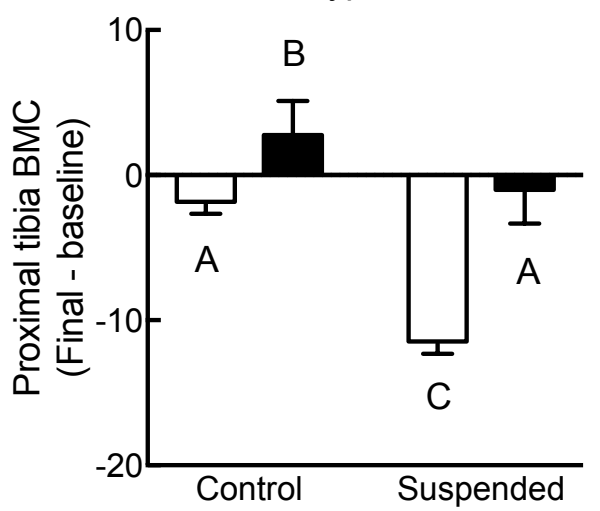

C.

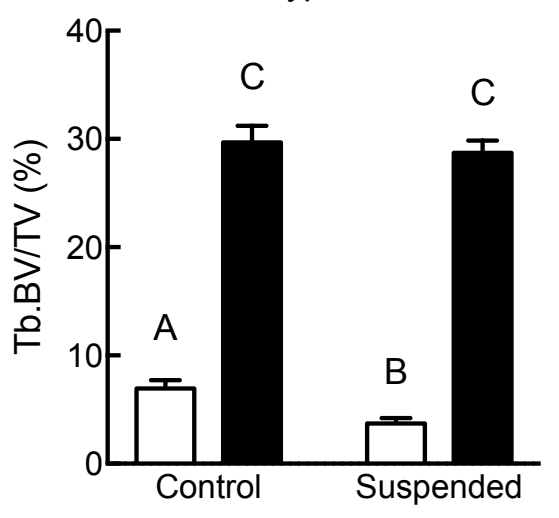

D. $\square$ Wild-type

Sost ${ }^{1-}$

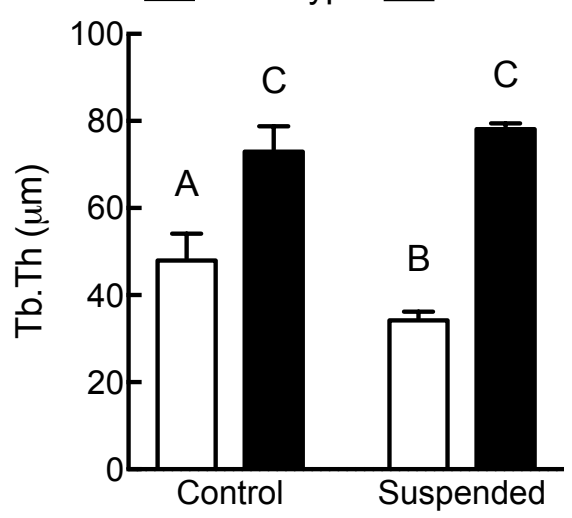

E. $\square$ Wild-type $\square$ Sost $^{\text {- }}$

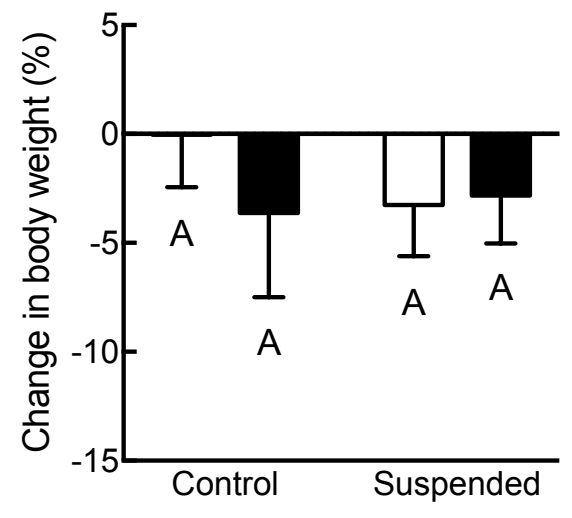


A Ground Control
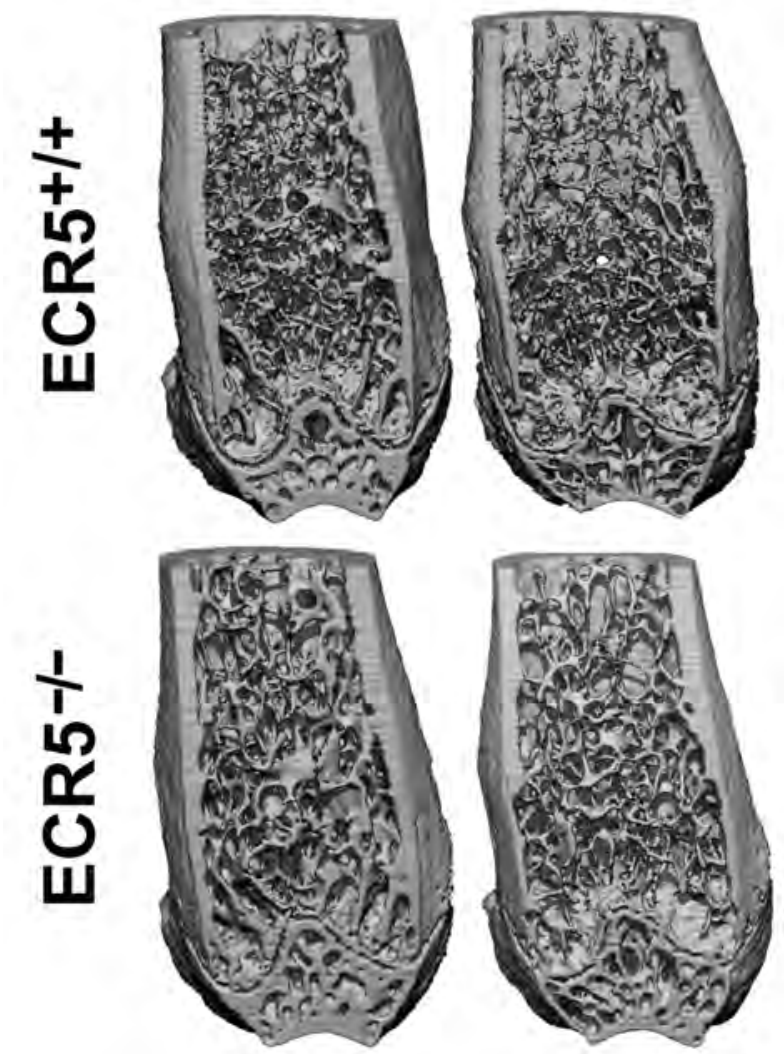

B.

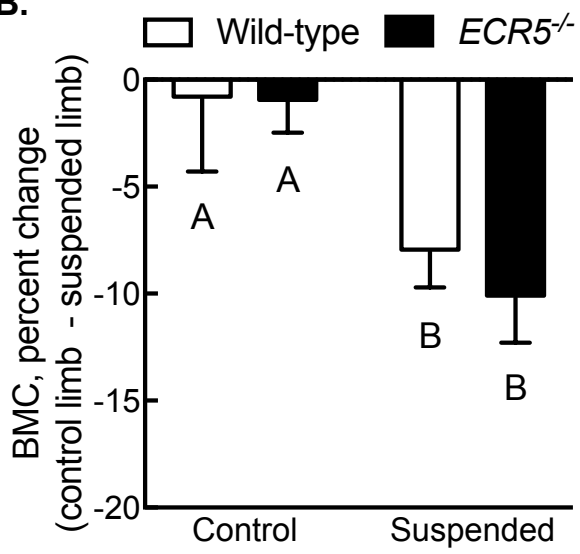

c.

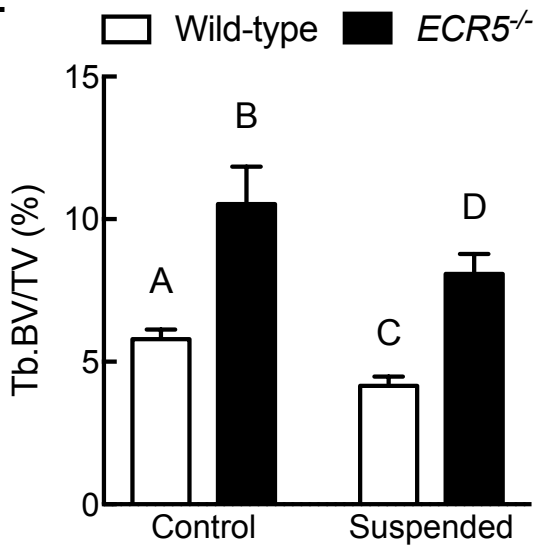

D.

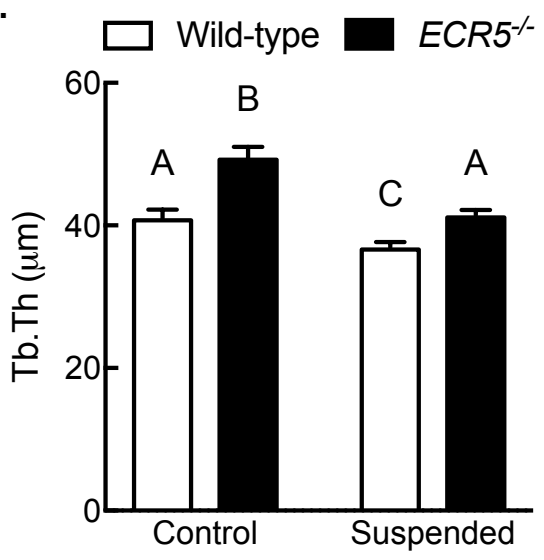

E.

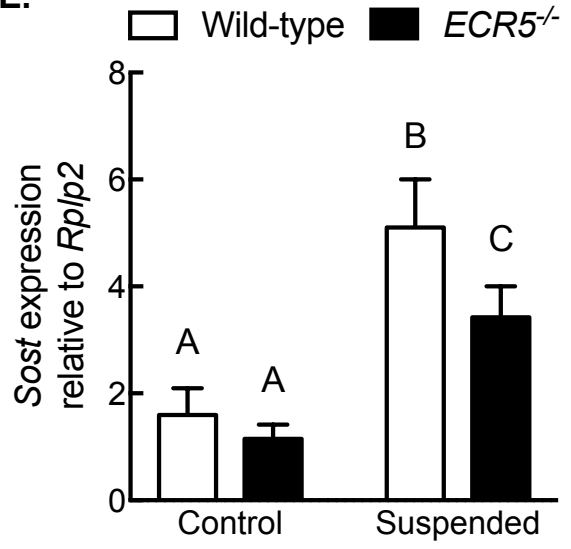

